\title{
On Hopf-Galois extensions of linear categories
}

\author{
Anca Stănescu
}

\begin{abstract}
We continue the investigation of $H$-Galois extensions of linear categories, where $H$ is a Hopf algebra. In our main result, the Theorem 2.2 , we characterize this class of extensions in the case when $H$ is finite dimensional. As an application, we prove a version of the Duality Theorem for crossed products with invertible cocycle.
\end{abstract}

\section{Introduction}

The duality theorems for actions and coactions originated in the work of Nagakami and Tagasaki on operator algebras, see [12]. A first purely algebraic version of the duality theorem was proved for actions of finite groups in [5]. Blattner and Montgomer showed that a similar result can be obtained for the actions and the coactions of a finite dimensional Hopf algebra, see [2]. A variant of the duality theorem for $\mathbb{k}$-linear graded categories was considered in [3] in order to investigate the Galois coverings of a linear category.

In this paper we continue our work on Hopf-Galois extensions of linear categories, started in [14]. Our main aim now is to prove a duality theorem for the crossed product of linear category with a finite dimensional Hopf algebra. Almost all of the contents of the article are the adaptation of the results known for algebras to the case of linear categories.

In the first part of the article we fix the terminology and the notation that we use. All the definitions of small linear categories and modules over them in Subsection 1.1, as well as the results on projective modules in Subsection 1.5

Key Words: Linear categories, Hopf-Galois extensions, Crossed products.

2010 Mathematics Subject Classification: Primary 18D20; Secondary 16T05.

Received: April, 2011.

Revised: April, 2011.

Accepted: February, 2012. 
are essentially due to B. Mitchell, who was one of the pioneers of regarding a small category as a ring with several objects (see [10]). Here we also recall the definition of the key concepts and the most important results which we obtained in the above mentioned paper.

Throughout, $H$ will denote a finite dimensional Hopf algebra over a field $\mathbb{k}$. In the second section, following closely the method of [11, Chapter VIII], we give some characterizations of $H$-Galois extensions of linear categories, see the Theorem 2.2. A key step in the proof of this result is the Theorem 2.1, which generalizes the well-known result due to Kreimer and Takeuchi [9], stating that any $H$-comodule algebra is $H$-Galois, provided that the canonical Galois map is surjective.

Given an $H$-Galois extension $\mathcal{D} \subseteq \mathcal{C}$ of linear categories, as a corollary of Theorem 2.2, we show that a certain functor $\pi: \mathcal{C} \# H^{*} \longrightarrow \mathcal{E}\left(\mathcal{C}_{\mathcal{D}}\right)$ is an isomorphism of linear categories, where $\mathcal{E}\left(\mathcal{C}_{\mathcal{D}}\right)$ is the linear category constructed in $\S 1.6$. If $\mathcal{C}$ is a crossed product with invertible 2 -cocycle $\mathcal{C} \simeq \mathcal{D} \#{ }_{\sigma} H$, then $\mathcal{E}\left(\mathcal{C}_{\mathcal{D}}\right)$ is isomorphic to the linear category $\mathcal{M}_{n}(\mathcal{D})$ of square matrices of order $n$ with elements in $\mathcal{D}$, where $n=\operatorname{dim} H$. As an application, we immediately get the Corollary 2.3, which is our version of the duality theorem.

\section{Preliminaries}

In this section we recall the main concepts needed in this paper. For this part the reader is referred to [14]. We begin by recalling the definition and the properties of Hopf-Galois extensions of linear categories.

1.1. H-Galois extensions of linear categories. A small $\mathbb{k}$-linear category is given by a set $\mathcal{C}_{0}$ and the vector spaces of morphisms from $y$ to $x$ in the category $\mathcal{C}$, denoted by ${ }_{x} \mathcal{C}_{y}$, for each $x, y \in \mathcal{C}_{0}$, together with the composition maps

$$
\circ:{ }_{x} \mathrm{e}_{y} \otimes{ }_{y} \mathrm{e}_{z} \longrightarrow{ }_{x} \mathrm{e}_{z}
$$

and the identity morphisms $1_{x} \in{ }_{x} \mathcal{C}_{x}$, for each $x \in \mathcal{C}_{0}$. These data satisfy the associativity axiom and the identity morphism axiom.

If $\mathcal{C}$ is a linear category, a left $\mathcal{C}$-module is given by a family of $\mathbb{k}$-vector spaces $\left\{{ }_{x} M\right\}_{x \in \mathcal{C}_{0}}$ together with the structure maps

$$
{ }_{x} \triangleright_{y}:{ }_{x} \mathcal{C}_{y} \otimes{ }_{y} M \longrightarrow{ }_{x} M
$$

which satisfy the equations (1) and (2) in [14]. The right $\mathcal{C}$-modules are defined similarly. A $(\mathcal{C}, \mathcal{D})$-bimodule is defined by a family $\left\{{ }_{x} M_{y}\right\}_{(x, y) \in \mathcal{C}_{0} \times \mathcal{D}_{0}}$ together with the maps

$$
{ }_{x} \triangleright_{y}^{u}:{ }_{x} \mathrm{C}_{u} \otimes{ }_{u} M_{y} \longrightarrow{ }_{x} M_{y} \quad \text { and } \quad{ }_{x} \triangleleft_{y}^{v}:{ }_{x} M_{v} \otimes{ }_{v} \mathcal{D}_{y} \longrightarrow{ }_{x} M_{y}
$$


such that $\left\{{ }_{u} M_{y}\right\}_{u \in \mathcal{C}_{0}}$ is a left $\mathcal{C}$-module with respect to $\left\{{ }_{x} \triangleright_{y}^{u}\right\}_{u \in \mathcal{C}_{0}}$ and, similarly, $\left\{{ }_{x} M_{v}\right\}_{v \in \mathcal{D}_{0}}$ is a right $\mathcal{D}$-module with respect to $\left\{{ }_{x} \triangleleft_{y}^{v}\right\}_{v \in \mathcal{D}_{0}}$, for any $x$ in $\mathcal{C}_{0}$ and $y$ in $\mathcal{D}_{0}$. These modules will be denoted by $\bullet M_{y}$ and ${ }_{x} M_{\bullet}$, respectively.

Any linear category $\mathcal{C}$ can be regarded as a bimodule over itself. The components of this bimodule are the spaces ${ }_{x} \mathcal{C}_{y}$, and the structure maps are given by the composition maps of $\mathcal{C}$. In particular, we can consider the right $\mathcal{C}$-module ${ }_{x} \mathcal{C}_{\bullet}$ and the left $\mathcal{C}$-module $\mathcal{C}_{y}$, for any $x, y \in \mathcal{C}_{0}$. If $M$ is a right $\mathcal{D}$-module and $N$ is a left $\mathcal{D}$-module one defines the $\mathbb{k}$-vector space $M \otimes_{\mathcal{D}} N$ as in $[14, \S 1.8]$.

For a Hopf algebra $H$ we shall say that a linear category $\mathcal{C}$ is a (right) $H$ comodule category, if every ${ }_{x} \mathrm{e}_{y}$ is a (right) $H$-comodule such that its structure map

$$
{ }_{x} \rho_{y}:{ }_{x} \mathcal{C}_{y} \longrightarrow{ }_{x} \mathcal{C}_{y} \otimes H
$$

satisfies the relations

$$
\begin{aligned}
{ }_{x} \rho_{z}\left(f \circ f^{\prime}\right) & =\sum f_{0} \circ f_{0}^{\prime} \otimes f_{1} f_{1}^{\prime}, \\
{ }_{x} \rho_{x}\left(1_{x}\right) & =1_{x} \otimes 1_{H},
\end{aligned}
$$

for any $f \in{ }_{x} \mathcal{C}_{u}$ and $f^{\prime} \in{ }_{u} \mathcal{C}_{y}$. In the above equations we used the Sweedler notation ${ }_{x} \rho_{y}:=\sum f_{\langle 0\rangle} \otimes f_{\langle 1\rangle}$.

If $H$ is finite dimensional, then the linear dual $H^{*}$ of $H$ is a Hopf algebra which acts on the spaces ${ }_{x} \mathcal{C}_{y}$ by the operations

$$
\cdot: H^{*} \otimes{ }_{x} \mathcal{e}_{y} \longrightarrow{ }_{x} \mathfrak{e}_{y}, \quad \alpha \cdot f=\sum \alpha\left(f_{1}\right) f_{0} .
$$

It is well know that $\mathcal{C}$ is an $H^{*}$-module category, i.e. we have

$$
\begin{aligned}
\alpha \cdot\left(f \circ f^{\prime}\right) & =\sum\left(\alpha_{1} \cdot f\right) \circ\left(\alpha_{2} \cdot f^{\prime}\right), \\
\alpha \cdot 1_{x} & =\alpha(1) 1_{x},
\end{aligned}
$$

for any $f$ and $f^{\prime}$ as above, $\alpha \in H^{*}$ and $x \in \mathcal{C}_{0}$.

For an $H$-comodule algebra $\mathcal{C}$ one defines the linear subcategory of $H$ coinvariant elements $\mathcal{C}^{c o H} \subseteq \mathcal{C}$ by $\mathcal{C}_{0}^{c o H}=\mathcal{C}_{0}$ and

$$
{ }_{x} \mathcal{C}_{y}^{c o H}:=\left\{f \in{ }_{x} \mathcal{C}_{y} \mid{ }_{x} \rho_{y}(f)=f \otimes 1_{H}\right\}, \forall x, y \in \mathcal{C}_{0} .
$$

For simplicity we shall use the notation $\mathcal{D}=\mathcal{C}^{c o H}$. Following [7], for all $x$ and $y$ in $\mathcal{C}_{0}$ and any (right) $H$-comodule category $\mathcal{D} \subseteq \mathcal{C}$, one defines the Galois canonical maps $\left\{{ }_{x} \beta_{y}\right\}_{x, y \in \mathcal{C}_{0}}$ by:

$$
{ }_{x} \beta_{y}:{ }_{x} \mathcal{C}_{\bullet} \otimes_{\mathcal{D}} \bullet \mathcal{C}_{y} \longrightarrow{ }_{x} \mathcal{C}_{y} \otimes H, \quad{ }_{x} \beta_{y}\left(f \otimes_{\mathcal{D}} f^{\prime}\right)=\sum f \circ f_{0}^{\prime} \otimes f_{1}^{\prime},
$$


where in the above formula $f \in{ }_{x} \mathrm{e}_{u}$ and $f^{\prime} \in{ }_{u} \mathrm{C}_{y}$. By definition, the extension $\mathcal{D} \subseteq \mathcal{C}$ is $H$-Galois if and only if ${ }_{x} \beta_{y}$ is bijective for all $x, y \in \mathcal{C}_{0}$.

Analogously, the maps $\left\{{ }_{x} \beta_{y}^{\prime}\right\}_{x, y \in \mathcal{C}_{0}}$ are defined by:

$$
{ }_{x} \beta_{y}^{\prime}:{ }_{x} \mathcal{C}_{\bullet} \otimes_{\mathcal{D}} \bullet \mathcal{C}_{y} \longrightarrow{ }_{x} \mathcal{C}_{y} \otimes H, \quad{ }_{x} \beta_{y}^{\prime}\left(f \otimes_{\mathcal{D}} f^{\prime}\right)=\sum f_{0} \circ f^{\prime} \otimes f_{1} .
$$

If the antipode of $H$ is invertible, proceeding as in [11, p. 124], one shows that the (right) $H$-comodule category $\mathcal{D} \subseteq \mathcal{C}$ is $H$-Galois if and only if ${ }_{x} \beta_{y}^{\prime}$ is bijective for all $x$ and $y$ in $\mathrm{C}_{0}$.

1.2. Crossed products. In [14] we have seen that the crossed product of a linear category $\mathcal{D}$ and a Hopf algebra $H$ is an $H$-Galois extension of linear categories. We recall that a crossed product is given by a weak action $\{x \cdot y\}_{x, y \in \mathcal{D}_{0}}$ of $H$ on $\mathcal{D}$ together with a normalized 2-cocycle $\sigma:=\left\{\sigma_{x}\right\}_{x \in \mathcal{D}_{0}}$ that satisfies the twisted module condition. The weak action is defined by the maps ${ }_{x}{ }_{y}: H \otimes{ }_{x} \mathcal{D}_{y} \longrightarrow{ }_{x} \mathcal{D}_{y}$ which satisfy the following conditions:

$$
\begin{aligned}
h_{x} \cdot z\left(f \circ f^{\prime}\right) & =\sum\left(h_{1 x \cdot y} f\right) \circ\left(h_{2 y \cdot z} f^{\prime}\right), \\
h_{x} \cdot{ }_{x} 1_{x} & =\varepsilon(h) 1_{x}, \\
1_{H x} \cdot{ }_{y} f & =f .
\end{aligned}
$$

For simplicity we shall write $h \cdot f$ instead of $h_{x} \cdot y$, for any $h \in H$ and $f \in{ }_{x} \mathcal{D}_{y}$.

On the other hand, the 2-cocycle $\sigma$ is given by the maps $\sigma_{x}: H \otimes H \longrightarrow{ }_{x} \mathcal{D}_{x}$ such that the following relation holds:

$$
\sum\left[h_{1} \cdot \sigma_{x}\left(h_{1}^{\prime}, h_{1}^{\prime \prime}\right)\right] \circ \sigma_{x}\left(h_{2}, h_{2}^{\prime} h_{2}^{\prime \prime}\right)=\sum \sigma_{x}\left(h_{1}, h_{1}^{\prime}\right) \circ \sigma_{x}\left(h_{2} h_{2}^{\prime}, h^{\prime \prime}\right) .
$$

The cocycle $\sigma$ is normalized if for $x \in \mathcal{D}_{0}$ and $h \in H$ we have:

$$
\sigma_{x}(h, 1)=\sigma_{x}(1, h)=\varepsilon(h) 1_{x},
$$

and the weak action of $H$ on $\mathcal{D}$ satisfies the twisted module condition if and only if, for $x, y \in \mathcal{D}_{0}, f \in{ }_{x} \mathcal{D}_{y}$ and $h, h^{\prime} \in H$,

$$
\sum\left[h_{1} \cdot\left(h_{1}^{\prime} \cdot f\right)\right] \circ \sigma_{y}\left(h_{2}, h_{2}^{\prime}\right)=\sum \sigma_{x}\left(h_{1}, h_{1}^{\prime}\right) \circ\left[\left(h_{2} h_{2}^{\prime}\right) \cdot f\right] .
$$

The crossed product $\mathcal{D} \#_{\sigma} H$ is the small linear category which is defined as follows. Its set of objects is $\mathcal{D}_{0}$ and ${ }_{x}\left(\mathcal{D} \#{ }_{\sigma} H\right)_{y}={ }_{x} \mathcal{D}_{y} \otimes H$. The identity maps are $1_{x} \otimes 1_{H}$ and the composition is given by

$$
\left.(f \# h)_{x} \circ z=f^{\prime} \# h^{\prime}\right)=\sum f \circ\left(h_{1} \cdot f^{\prime}\right) \circ \sigma_{z}\left(h_{2}, h_{1}^{\prime}\right) \# h_{3} h_{2}^{\prime} .
$$

There is an obvious linear functor $\mathcal{D} \longrightarrow \mathcal{D} \#_{\sigma} H$, which is the identity on objects and sends $f$ to $f \# 1_{H}$. This allows to regard a $\mathcal{D} \#{ }_{\sigma} H$-module as a 
$\mathcal{D}$-module. Let $\mathcal{C}:=\mathcal{D} \#{ }_{\sigma} H$. By construction $\mathcal{D} \subseteq \mathcal{C}$ has the normal basis property, i.e. there is an isomorphism $\bullet \mathcal{C}_{x} \simeq \bullet^{\mathcal{D}} \mathcal{D}_{x} \otimes H$ of left $\mathcal{D}$-modules and right $H$-comodules, for all $x \in \mathcal{C}_{0}$. In particular this means that $\bullet \mathcal{C}_{x}$ is a free left $\mathcal{D}$-module, for all $x \in \mathcal{C}_{0}$.

Note that, in the case when $H$ is finite dimensional, the left $\mathcal{D}$-module - $\mathcal{C}_{x}$ is also finitely generated. If, in addition, the 2-cocycle $\sigma$ is invertible in convolution then the right $\mathcal{D}$-module ${ }_{x} \mathcal{C}_{\bullet}$ is free and finitely generated as well. As a matter of fact, under these assumptions, ${ }_{x} \mathrm{C}_{\bullet}$ is isomorphic to ${ }_{x} \mathcal{D} \bullet \otimes H$, for any $x \in \mathcal{C}_{0}$. By hypothesis, the antipode $S$ of $H$ is bijective. Let $\bar{S}$ denote its inverse. Proceeding as in the proof of [6, Proposition 6.1.10(iv)] one can show that the maps

${ }_{x} \alpha_{y}: H \otimes{ }_{x} \mathcal{D}_{y} \longrightarrow{ }_{x} \mathcal{D}_{y} \# H, \quad{ }_{x} \alpha_{y}(h \otimes f)=\sum \sigma_{x}^{-1}\left(h_{2}, \bar{S} h_{1}\right) \circ\left(h_{3} \cdot f\right) \otimes h_{4}$

${ }_{x} \beta_{y}:{ }_{x} \mathcal{D}_{y} \# H \longrightarrow H \otimes{ }_{x} \mathcal{D}_{y}, \quad{ }_{x} \beta_{y}(f \otimes h)=\sum h_{4} \otimes\left(\bar{S} h_{3} \cdot f\right) \circ \sigma_{y}\left(\bar{S} h_{2}, h_{1}\right)$

define morphisms of right $\mathcal{D}$-modules

$$
{ }_{x} \alpha_{\bullet}: H \otimes{ }_{x} \mathcal{D} \bullet \longrightarrow{ }_{x} \mathcal{D} \bullet \# H \text { and }{ }_{x} \beta_{\bullet}:{ }_{x} \mathcal{D} \bullet \# H \longrightarrow H \otimes{ }_{x} \mathcal{D} \bullet
$$

which are inverses of each other. In particular, we deduce that ${ }_{x} \mathcal{C}_{\bullet}$ is isomorphic as a right $\mathcal{D}$-module with ${ }_{x} \mathcal{D}_{\bullet}^{n}$, where $n=\operatorname{dim} H$. Here ${ }_{x} \mathcal{D}_{\bullet}^{n}$ is the $\mathcal{D}$-module whose components are the $\mathbb{k}$-linear spaces ${ }_{x} \mathcal{D}_{y}^{n}={ }_{x} \mathcal{D}_{y} \oplus \ldots \oplus{ }_{x} \mathcal{D}_{y}$ ( $n$ summands), and the module structure is given by the composition maps of D.

1.3. Hopf modules over finitely dimensional Hopf algebras. We recall that if $\mathcal{D} \subseteq \mathcal{C}$ is an $H$-comodule category, a left $\mathcal{C}$-module $(M, \triangleright)$ is called Hopf e-module if $H$ coacts on $M$ such that

$$
\rho(f \triangleright m)=\sum f_{0} \triangleright m_{0} \otimes f_{1} m_{1} .
$$

If $H$ is finite dimensional then the component ${ }_{x} M$ of $M$, for all $x \in \mathcal{C}_{0}$, is a left $H^{*}$-module with respect to the action

$$
\alpha \cdot m=\sum \alpha\left(m_{1}\right) m_{0} .
$$

To give a Hopf module $(M, \triangleright, \cdot)$ is equivalent to give a $\mathcal{C} \# H^{*}$-action $(M, \rightarrow)$. These structures are related by the formula:

$$
(f \# \alpha) \rightarrow m=f \triangleright(\alpha \cdot m),
$$

where $f \in{ }_{x} \mathcal{C}_{y}, m \in{ }_{y} M$ and $\alpha \in H^{*}$. This operation defines a $\mathcal{C} \# H^{*}$-module structure because, for any $\alpha, f$ and $m$ as above,

$$
\alpha \cdot(f \triangleright m)=\sum\left(\alpha_{1} \cdot f\right) \triangleright\left(\alpha_{2} \cdot m\right) .
$$


Remark that for any left Hopf module $M$ we have $\left({ }_{x} M\right)^{\operatorname{co}(H)}=\left({ }_{x} M\right)^{H^{*}}$.

If $\lambda \in H^{*}$ is a left integral, then

$$
\left(1_{x} \# \lambda\right) \rightarrow m=1_{x} \triangleright(\lambda \cdot m)=\lambda \cdot m .
$$

Therefore, for any $\alpha \in H^{*}$ and $m \in{ }_{x} M$ we have

$\alpha \cdot\left[\left(1_{x} \# \lambda\right) \rightarrow m\right]=\alpha \cdot(\lambda \cdot m)=(\alpha * \lambda) \cdot m=\alpha(1) \lambda \cdot m=\alpha(1)\left[\left(1_{x} \# \lambda\right) \rightarrow m\right]$,

where the product of $H^{*}$ is denoted by $*$. In conclusion $\left(1_{x} \otimes \lambda\right) \rightarrow m$ is an element of $\left({ }_{x} M\right)^{H^{*}}$. In other words, this element is $H$-coinvariant. Since $\bullet \mathcal{C}_{y}$ is a left Hopf module for any $y \in \mathcal{C}_{0}$, we deduce in particular that

$$
(1 \# \lambda) \rightarrow f \in{ }_{x} \mathcal{C}_{y}^{c o(H)}={ }_{x} \mathcal{D}_{y},
$$

for any $f \in \in_{x} \mathcal{C}_{y}$. Let us notice that the $\mathcal{C} \# H^{*}$-action $(M, \rightarrow)$ and the initial $\mathcal{C}$ action $(M, \triangleright)$ are compatible in the sense that, for any $f \in{ }_{x} \mathcal{C}_{y}, z \in{ }_{y}\left(\mathcal{C} \# H^{*}\right)_{u}$ and $m \in{ }_{u} M$,

$$
f \triangleright(z \cdot m)=[(f \# \varepsilon) z] \rightarrow m .
$$

Equivalently, the $\mathcal{C}$-module $(M, \triangleright)$ is recovered from the $\mathcal{C} \# H^{*}$-module $(M, \rightarrow)$ by the restriction of scalars via the inclusion functor $\mathcal{C} \hookrightarrow \mathcal{C} \# H^{*}$, which is the identity function on the set of objects and maps any $f$ in ${ }_{x} \mathrm{e}_{y}$ to $f \otimes \varepsilon$.

1.4. The diagonal action. Let $H$ be a Hopf algebra over a field $\mathbb{k}$. If $V$ and $W$ are left $H$-modules then $V \otimes W$ is a left $H$-module via the diagonal action, which is defined by

$$
h \cdot(v \otimes w)=\sum\left(h_{1} \cdot v\right) \otimes\left(h_{2} \cdot w\right),
$$

for all $h \in H, v \in V$ and $w \in W$. In particular, $V \otimes H$ is a left $H$-module with respect to the diagonal action.

On the other hand $H \otimes V$ is a left module with respect to the action

$$
h \cdot(k \otimes v)=(h k) \otimes v .
$$

If the antipode $S$ of $H$ is bijective, then $\mu: H \otimes V \rightarrow V \otimes H$ which maps $h \otimes v$ to $\sum h_{1} \cdot v \otimes h_{2}$ is an isomorphism of left $H$-modules. Indeed, the map $v \otimes h \mapsto \sum h_{2} \otimes \bar{S} h_{1} \cdot v$ is an inverse of $\mu$, where $\bar{S}$ denotes the inverse of $S$. The map $\mu$ induces an $\mathbb{k}$-linear isomorphism between the set of invariant elements in $V \otimes H$ and $H \otimes V$. In the case when $H$ is finite dimensional, we have

$$
(H \otimes V)^{H}=H^{H} \otimes V=\mathbb{k} t \otimes V,
$$

where $t$ is a left integral in $H$. It follows that $(V \otimes H)^{H}=\left\{\sum t_{1} \cdot v \otimes t_{2} \mid v \in V\right\}$, as any invariant element in $V \otimes H$ can be written as $\mu(t \otimes v)$, for some $v \in V$. 
1.5. Projective $\mathcal{D}$-modules. Let $\mathcal{D}$ be a linear category. A right $\mathcal{D}$-module $M$ is projective and finitely generated if it is a direct sumand of a module of the form $\bigoplus_{i=1}^{n} x_{i} \mathcal{D}$. where $x_{1}, \ldots, x_{n}$ are some objects in $\mathcal{D}_{0}$. Indeed, by definition, a right $\mathcal{D}$-module $M$ is finitely generated if and only if there exist $x_{1}, \ldots, x_{n} \in \mathcal{D}_{0}$ together with an epimorphism

$$
\pi: \bigoplus_{i=1}^{n} x_{i} \mathcal{D} \bullet \longrightarrow M
$$

Since $M$ is projective the map $\pi$ splits, i.e. there is a section $\sigma$ of $\pi$ in the category of right $\mathcal{D}$-modules. Thus $M$ is a direct summand of $\bigoplus_{i=1}^{n} x_{i} \mathcal{D}$.

Conversely, let us assume that $M$ is a direct summand in $X=\bigoplus_{i=1}^{n_{2} x_{i}} \mathcal{D}$. Clearly, a direct summand of a projective module is projective too, and an arbitrary direct sum of projective modules is projective. Therefore, in order to prove that $M$ is projective, it is enough to show that ${ }_{x} \mathcal{D}$. is projective, for any $x \in \mathcal{D}_{0}$. This property immediately follows from the definition of projective $\mathcal{D}$-modules.

We also need the following characterization of finitely generated projective modules: the right $\mathcal{D}$-module $(M, \triangleleft)$ is projective and finitely generated if and only if there exist a finite number of objects $x_{1}, \ldots, x_{n} \in \mathcal{D}_{0}$, elements $m^{i} \in M_{x_{i}}$ and $\mathcal{D}$-linear morphisms $\varphi^{i}: M \longrightarrow{ }_{x_{i}} \mathcal{D} \bullet$ such that, for any $m \in M_{x}$ we have:

$$
m=\sum_{i=1}^{n} m^{i} \triangleleft \varphi_{x}^{i}(m) .
$$

Here, of course, $\varphi_{x}^{i}:{ }_{x_{i}} \mathcal{D}_{x} \longrightarrow M_{x}$ denotes the $x$-component of $\varphi^{i}$.

To prove this characterization let us note that $M$ is projective and finitely generated if and only if there is an epimorphism $\pi: \bigoplus_{i=1}^{n} x_{i} \mathcal{D} \bullet \longrightarrow M$ that splits in the category of right $\mathcal{D}$-modules. Let $\sigma$ be a section of $\pi$. We define

$$
m^{i}=\pi_{x_{i}}\left(0, \ldots, \operatorname{Id}_{x_{i}}, \ldots, 0\right) \in M_{x_{i}}
$$

and we denote by $p^{i}: \bigoplus_{j=1}^{n} x_{j} \mathcal{D} \bullet \longrightarrow x_{i} \mathcal{D}$. the canonical projection. If we take $\varphi^{i}:=\sigma \circ p^{i}$, then one can easily see that the equation (3) holds for any $m \in M_{x}$.

Conversely, let $x_{1}, \ldots, x_{n} \in \mathcal{D}_{0}$. We assume that there are the elements $m^{i} \in M_{x_{i}}$ and the morphisms $\varphi^{i}:=M \longrightarrow{ }_{x_{i}} \mathcal{D}$ such that (3) holds. We define

$$
\begin{gathered}
\pi_{x}: \bigoplus_{i=1}^{n} x_{i} \mathcal{D}_{x} \longrightarrow M_{x}, \quad \pi_{x}\left(f_{1}, \ldots, f_{n}\right)=\sum_{i=1}^{n} m_{i} \triangleleft f_{i}, \\
\sigma_{x}: M_{x} \longrightarrow \bigoplus_{i=1}^{n} x_{i} \mathcal{D}_{x}, \quad \sigma_{x}(m)=\left(\varphi_{x}^{1}(m), \ldots, \varphi_{x}^{n}(m)\right) .
\end{gathered}
$$


Obviously $\pi=\left\{\pi_{x}\right\}_{x \in \mathcal{C}_{0}}$ and $\sigma=\left\{\sigma_{x}\right\}_{x \in \mathcal{C}_{0}}$ are morphisms of $\mathcal{D}$-modules and $\sigma$ is a section for $\pi$.

1.6. The category $\mathcal{E}\left(M_{\mathcal{D}}\right)$. Let $\mathcal{C}$ and $\mathcal{D}$ be two $\mathbb{k}$-linear categories such that $\mathcal{C}_{0}=\mathcal{D}_{0}=S$. Let $(M, \triangleright, \triangleleft)$ be a $(\mathcal{C}, \mathcal{D})$-bimodule with components $\left\{{ }_{x} M_{y}\right\}_{x, y \in S}$. We define the $\mathbb{k}$-linear category $\mathcal{E}\left(M_{\mathcal{D}}\right)$ such that $\mathcal{E}\left(M_{\mathcal{D}}\right)_{0}=S$. By definition,

$$
{ }_{x} \mathcal{E}\left(M_{\mathcal{D}}\right)_{y}=\operatorname{Hom}_{\mathcal{D}}\left({ }_{y} M_{\bullet},{ }_{x} M_{\bullet}\right) .
$$

Since ${ }_{y} M_{\bullet}$ is the right $\mathcal{D}$-module having the components $\left\{{ }_{y} M_{u}\right\}_{u \in S}$, a morphism in ${ }_{x} \mathcal{E}\left(M_{\mathcal{D}}\right)_{y}$ is a family $f:=\left\{f_{u}\right\}_{u \in S}$ of $\mathbb{k}$-linear maps $f_{u}:{ }_{y} M_{u} \longrightarrow$ ${ }_{x} M_{u}$, which defines a morphism of right $\mathcal{D}$-modules $f:{ }_{y} M_{\bullet} \longrightarrow{ }_{x} M_{\bullet}$. The composition in $\mathcal{E}\left(M_{\mathcal{D}}\right)$ is given by

$\circ:{ }_{x} \mathcal{E}\left(M_{\mathcal{D}}\right)_{y} \otimes_{y} \mathcal{E}\left(M_{\mathcal{D}}\right)_{z} \longrightarrow{ }_{x} \mathcal{E}\left(M_{\mathcal{D}}\right)_{z}, \quad\left\{f_{u}\right\}_{u \in S} \circ\left\{g_{u}\right\}_{u \in S}=\left\{f_{u} \circ g_{u}\right\}_{u \in S}$

and the identity morphism of $x$ is the family $\left\{\operatorname{Id}_{x} M_{u}\right\}_{u \in S}$.

The $\mathbb{k}$-linear category $\mathcal{E}(\mathrm{e} M)$ is similarly constructed. We set $\mathcal{E}(\mathrm{e} M)_{0}=S$ and

$$
{ }_{x} \mathcal{E}(\mathrm{e} M)_{y}=\operatorname{Hom}_{\mathcal{C}}\left(\bullet M_{y}, \bullet M_{x}\right) .
$$

Since $\bullet M_{y}$ is the left $\mathcal{C}$-module having the components $\left\{{ }_{u} M_{y}\right\}_{u \in S}$, a morphism in ${ }_{x} \mathcal{E}(\mathrm{e} M)_{y}$ is a family $f:=\left\{f_{u}\right\}_{u \in S}$ of $\mathbb{k}$-linear maps $f_{u}:{ }_{u} M_{y} \longrightarrow{ }_{u} M_{x}$, which defines a morphism of right $\mathcal{D}$-modules $f:{ }_{y} M_{\bullet} \longrightarrow{ }_{x} M_{\bullet}$. The composition in $\mathcal{E}(e M)$ is given by

$\circ:{ }_{x} \mathcal{E}(e M)_{y} \otimes_{y} \mathcal{E}(e M)_{z} \longrightarrow{ }_{x} \mathcal{E}(e M)_{y}, \quad\left\{f_{u}\right\}_{u \in S} \circ\left\{g_{u}\right\}_{u \in S}=\left\{f_{u} \circ g_{u}\right\}_{u \in S}$

and the identity morphism of $x$ is the family $\left\{\operatorname{Id}_{x} M_{u}\right\}_{u \in S}$.

For any $(\mathcal{C}, \mathcal{D})$-bimodule $M$ there exists a $\mathbb{k}$-linear functor $\Pi^{\mathcal{C}}: \mathcal{C} \longrightarrow$ $\mathcal{E}\left(M_{\mathcal{D}}\right)$, such that $\Pi^{\mathcal{C}}$ maps every object of $\mathcal{C}$ to itself. Moreover, for $f \in{ }_{x} \mathcal{C}_{y}$ we define ${ }_{x} \Pi_{y}^{\mathcal{C}}(f):{ }_{y} M_{\bullet} \longrightarrow{ }_{x} M_{\bullet}$ to be the morphism of right $\mathcal{D}$-modules whose $u$-component maps $m \in{ }_{y} M_{u}$ to $f \triangleright m$, for any $u \in S$. Let us note that the above definition makes sense as $f \triangleright m \in{ }_{x} M_{u}$ for any $m \in{ }_{y} M_{u}$. One can easily see that in this way we obtain indeed a functor $\Pi^{\mathcal{C}}: \mathcal{C} \longrightarrow \mathcal{E}\left(M_{\mathcal{D}}\right)$.

The $\mathbb{k}$-linear functor $\Pi^{\mathcal{D}}: \mathcal{D}^{o p} \longrightarrow \mathcal{E}\left(e_{e} M\right)$ is defined in a similar way, where $\mathcal{D}^{o p}$ is the opposite category of $\mathcal{D}$. By definition, $\Pi^{\mathcal{D}}(x)=x$, for any $x \in S$. For $g \in{ }_{x} \mathcal{D}_{y}^{o p}$ the $u$-component of ${ }_{x} \Pi_{y}^{\mathcal{D}}(g)$ maps $m \in{ }_{u} M_{y}$ to $m \triangleleft g$. Let us notice that $g$ is an element in ${ }_{x} \mathcal{D}_{y}^{o p}={ }_{y} \mathcal{D}_{x}$, therefore $m \triangleleft g \in{ }_{u} M_{x}$. In conclusion ${ }_{x} \Pi_{y}^{\mathcal{D}}(g)$ is well defined.

1.7. The category $M_{n}(\mathcal{D})$. Let us consider the $(\mathcal{D}, \mathcal{D})$-bimodule $M=V \otimes \mathcal{D}$, where $V$ is a $\mathbb{k}$-vector space. The components of $M$ are ${ }_{x} M_{y}=V \otimes_{x} \mathcal{D}_{y}$, and the left and the right module actions are defined by

$$
g^{\prime} \triangleright(z \otimes f) \triangleleft g^{\prime \prime}=z \otimes\left(g^{\prime} \circ f \circ g^{\prime \prime}\right),
$$


for any $f \in{ }_{x} \mathcal{D}_{y}, g^{\prime} \in{ }_{u} \mathcal{D}_{x}, g^{\prime \prime} \in{ }_{y} \mathcal{D}_{v}$ and $z \in V$. If $\left\{e_{1}, \ldots, e_{n}\right\}$ is a basis on $V$ then an element $m$ in ${ }_{x} M_{u}$ can be uniquely written as a sum

$$
m=\sum_{i=1}^{n} e_{i} \otimes f_{i},
$$

with $f_{i} \in{ }_{x} \mathcal{D}_{u}$ for any $i$. Therefore, if $\varphi: V \otimes_{y} \mathcal{D}_{\bullet} \longrightarrow V \otimes_{x} \mathcal{D}_{\bullet}$ is a morphism of right $\mathcal{D}$-modules, then

$$
\varphi_{x}\left(e_{i} \otimes 1_{y}\right)=\sum_{j=1}^{n} e_{j} \otimes f_{j i},
$$

where $f_{j i} \in{ }_{x} \mathcal{D}_{y}$. Hence, for $f_{1}, \ldots, f_{n} \in{ }_{y} \mathcal{D}_{u}$, it follows that we have:

$$
\varphi_{u}\left(\sum_{i=1}^{n} e_{i} \otimes f_{i}\right)=\sum_{i, j=1}^{n} e_{j} \otimes f_{j i} \circ f_{i} .
$$

This relation shows us that $\varphi$ is uniquely determined by the "matrix" $M_{n}(\varphi)$ whose $(i, j)$-entry is $f_{i j} \in{ }_{x} \mathcal{D}_{y}$, for any $1 \leq i, j \leq n$. One can easily see that

$$
M_{n}(\varphi \circ \psi)=M_{n}(\varphi) \circ M_{n}(\psi)
$$

and that $M_{n}\left(\operatorname{Id}_{V \otimes_{x} \mathcal{D}_{x}}\right)=I_{x}$, where $I_{x}$ is the "unit matrix" whose $(i, j)$-entry is $1_{x} \in{ }_{x} \mathcal{D}_{x}$ for $i=j$, and 0 for $i \neq j$.

From the foregoing discussion it follows that we can identify $\mathcal{E}\left((V \otimes \mathcal{D})_{\mathcal{D}}\right)$ with the $\mathbb{k}$-linear category $M_{n}(\mathcal{D})$ having the same objects as $\mathcal{D}$, and whose hom-spaces are ${ }_{x} M_{n}(\mathcal{D})_{y}:=M_{n}\left({ }_{x} \mathcal{D}_{y}\right)$. The identity of $x$ in $M_{n}(\mathcal{D})$ is the unit matrix $I_{x}$. The composition of the matrices $\left(f_{i j}\right)_{i, j} \in M_{n}\left({ }_{x} \mathcal{D}_{y}\right)$ and $\left(g_{i j}\right)_{i, j} \in$ $M_{n}\left({ }_{y} \mathcal{D}_{z}\right)$ is the matrix $\left(h_{i j}\right)_{i, j} \in M_{n}\left({ }_{x} \mathcal{D}_{z}\right)$, where

$$
h_{i j}=\sum_{k=1}^{n} f_{i j} \circ g_{j k} .
$$

\section{The main results}

In this section we prove our main results. We start by showing that the well known Kreimer-Takeuchi Theorem holds true for comodule categories as well. The proof of the Theorem 2.1 follows the lines of the proof given by H.-J. Schneider in [11, Chapter VIII], Theorem 3.1. The proof of Theorem 2.2 also follows the lines of the algebra case, using a straightforward generalization of a result of J. Bergen and S. Montgomery in [1], Lemma 2.5, and the obvious generalization of Morita theory for small linear categories (e.g. [7], Cor. 2.11, etc). Moreover, the proof of Theorem 2.2 follows the lines of the proof of Theorem 2.1 in [4]. 
Theorem 2.1. Let $H$ be a finite dimensional Hopf algebra. If $\mathcal{D} \subseteq \mathcal{C}$ is an $H$-comodule category such that the components of the canonical Galois map

$$
\beta: \mathcal{C} \otimes_{\mathcal{D}} \mathcal{C} \longrightarrow \mathcal{C} \otimes H
$$

are surjective, then $\mathcal{D} \subseteq \mathcal{C}$ is $H$-Galois and ${ }_{x} \mathcal{C}_{\bullet}$ is a projective right $\mathcal{D}$-module, for any $x \in \mathcal{C}_{0}$.

Proof. We first show that, for a given $x$, the right $\mathcal{D}$-module ${ }_{x} \mathrm{e}_{\bullet}$ is projective. For, we shall prove that there exist some objects $x_{1}, \ldots, x_{n} \in \mathcal{C}_{0}$, together with the elements $f^{i} \in{ }_{x} \mathcal{C}_{x_{i}}$ and the morphisms of right $\mathcal{D}$-modules $\varphi^{i}:{ }_{x} \mathcal{C}_{\bullet} \longrightarrow$ ${ }_{x_{i}} \mathcal{D}$., such that for any $f \in{ }_{x_{i}} \mathrm{e}_{u}$ we have

$$
f=\sum_{i=1}^{n} f^{i} \circ \varphi_{u}^{i}(f)
$$

Since $H$ is finite dimensional there exists a left integral $\lambda \in H^{*}$. Then

$$
\theta: H \longrightarrow H^{*}, \quad \theta(h)=\lambda<h
$$

is a $\mathbb{k}$-linear isomorphism, where $(\alpha<h)(k)=\alpha(h k)$, for any $\alpha \in H^{*}$ and $h, k \in H$. Thus, there is an unique $t \in H$ such that $\lambda<t=\varepsilon$. Since the components of $\beta$ are surjective maps, there exist $f^{i} \in{ }_{x} \mathrm{e}_{x_{i}}$ and $g^{i} \in{ }_{x_{i}} \mathrm{e}_{x}$ such that

$$
{ }_{x} \beta_{x}\left(\sum_{i=1}^{n} f^{i} \otimes_{\mathcal{D}} g^{i}\right)=1_{x} \# t .
$$

Let us notice that $\mathcal{D} \subseteq \mathcal{C}$ is an $H^{*}$-module category, because $H$ is finite dimensional. The action of $H^{*}$ on ${ }_{x} \mathfrak{C}_{y}$ is given by the formula

$$
\alpha \cdot f=\sum \alpha\left(f_{1}\right) f_{0}
$$

for any $\alpha \in H^{*}$ and $f \in{ }_{x} \mathfrak{C}_{y}$. Clearly, by definition, we have ${ }_{x} \mathfrak{e}_{y}^{H^{*}}={ }_{x} \mathfrak{e}_{y}^{c o(H)}$, where the linear space of $H^{*}$-invariant elements is given by

$$
{ }_{x} \mathcal{C}_{y}^{H^{*}}=\left\{f \in{ }_{x} \mathcal{C}_{y} \mid \alpha \cdot f=\alpha(1) f, \forall \alpha \in H^{*}\right\} .
$$

For any $f \in{ }_{x} \mathcal{C}_{u}$, we now define $\varphi_{u}^{i}:{ }_{x} \mathcal{C}_{u} \longrightarrow{ }_{x_{i}} \mathcal{D}_{u}$ by

$$
\varphi_{u}^{i}(f)=\lambda \cdot\left(g^{i} \circ f\right) .
$$

Let us notice that $\lambda \cdot f^{\prime}$ is a morphism in ${ }_{x} \mathcal{D}_{y}$ for any $f^{\prime} \in{ }_{x} \mathcal{C}_{y}$, because $\lambda$ is a left integral in $H^{*}$, that is $\alpha * \lambda=\alpha\left(1_{H}\right) \lambda$, for any $\alpha \in H^{*}$. 
We can now prove that the equality (4) is true, i.e. ${ }_{x} \mathrm{e} \bullet$ is a projective $\mathcal{D}$-module.

$$
\begin{aligned}
\sum f^{i} \circ \varphi_{u}^{i}(f) & =\sum_{i=1}^{n} f^{i} \circ \lambda \cdot\left(g^{i} \circ f\right) \\
& =\sum_{i=1}^{n} \sum f^{i} \circ\left(\lambda_{1} \circ g^{i}\right) \circ\left(\lambda_{2} \cdot f\right) \\
& =\sum_{i=1}^{n} \lambda_{1}\left(g_{1}^{i}\right) f^{i} \circ g_{0}^{i} \circ\left(\lambda_{2} \cdot f\right) \\
& =\sum_{i=1}^{n} \sum \lambda_{1}(t) 1_{x} \circ\left(\lambda_{2} \cdot f\right) \\
& =\sum_{i=1}^{n} \sum \lambda_{1}(t) \lambda_{2} \cdot f .
\end{aligned}
$$

Let us shortly explain how the above equalities have been obtained. The first one is obvious, by the definition of the morphisms $\varphi_{u}^{i}$. The second one results by the fact that $\mathcal{D} \subseteq \mathcal{C}$ is an $H^{*}$-module category, and for the third identity we used the definition of the element $\lambda_{1} \cdot g^{i}$. The fourth identity is an immediate consequence of the relation $\sum_{i=1}^{n} \lambda_{1}(t) f^{i} \circ g_{0}^{i}=\lambda_{1}(t) 1_{x}$, which in turn follows by (5). On the other hand,

$$
\left(\sum \lambda_{1}(t) \lambda_{2}\right)(x)=\sum \lambda_{1}(t) \lambda_{2}(x)=\lambda(t x)=(\lambda<t)(x) .
$$

Thus, in view of the foregoing computations and of the fact that $\lambda<t=\varepsilon$ we get

$$
\sum_{i=1}^{n} f_{i} \circ \varphi_{u}^{i}(f)=\sum \lambda_{1}(t) \lambda_{2} \cdot f=(\lambda\llcorner t) \cdot f=\varepsilon \cdot f=f .
$$

It remains to prove that the components of $\beta$ are injective. Since $H$ is finite dimensional we can use $\beta^{\prime}$ instead of $\beta$, see the last paragraph of the subsection §1.1. Let us choose an element $\xi:=\sum_{j=1}^{m} f_{j}^{\prime} \underset{\mathcal{D}}{\otimes} g_{j}^{\prime}$ in $\operatorname{Ker}_{x} \beta_{y}^{\prime}$, where $f_{j}^{\prime} \in{ }_{x} \mathcal{C}_{u_{j}}$ and $g_{j}^{\prime} \in{ }_{u_{j}} \mathrm{C}_{y}$. By (4) and the fact that $\varphi_{u_{j}}^{i}\left(f_{j}^{\prime}\right) \in{ }_{x_{i}} \mathcal{D}_{u_{j}}$ we get

$$
\xi=\sum_{j=1}^{m} \sum_{i=1}^{n} f_{i} \circ \varphi_{u_{j}}^{i}\left(f_{j}^{\prime}\right) \otimes_{\mathcal{D}} g_{j}^{\prime}=\sum_{i=1}^{n} f_{i} \otimes_{\mathcal{D}} \sum_{j=1}^{m} \varphi_{u_{j}}^{i}\left(f_{j}^{\prime}\right) \circ g_{j}^{\prime} .
$$

Moreover, by the construction of $\varphi^{i}$ and the definition of the $H^{*}$-action on 
${ }_{x_{i}} \mathrm{C}_{u_{j}}$ we have

$$
\begin{aligned}
\xi & =\sum_{i=1}^{n} f_{i} \otimes_{\mathcal{D}} \sum_{j=1}^{m}\left[\lambda \cdot\left(g^{i} \circ f_{j}^{\prime}\right)\right] \circ g_{j}^{\prime} \\
& =\sum_{i=1}^{n} f_{i} \otimes_{\mathcal{D}} \sum_{j=1}^{m} \sum \lambda_{1}\left(g_{1}^{i}\right) \lambda_{2}\left(\left(f_{j}^{\prime}\right)_{1}\right) g_{0}^{i} \circ\left(f_{j}^{\prime}\right)_{0} \circ g_{j}^{\prime} .
\end{aligned}
$$

Since $\xi$ is in the kernel of ${ }_{x} \beta_{y}^{\prime}$ we deduce that $\xi=0$, that is the components of $\beta^{\prime}$ are injective.

Let $\mathcal{D} \subseteq \mathcal{C}$ be an $H$-comodule category, where $H$ is finite dimensional. Recall that $\mathcal{C}$ is an $\left(\mathcal{C} \# H^{*}, \mathcal{D}\right)$-bimodule, so we can consider the functor

$$
\Pi^{\mathcal{C} \# H^{*}}: \mathcal{C} \# H^{*} \longrightarrow \mathcal{E}\left(\mathcal{C}_{\mathcal{D}}\right), \quad \Pi^{\mathcal{C} \# H^{*}}(f \# \alpha)\left(f^{\prime}\right)=f \circ\left(\alpha \cdot f^{\prime}\right),
$$

where $f \in{ }_{x} \mathcal{C}_{u}$ and $f^{\prime} \in{ }_{u} \mathcal{C}_{y}$, and $\alpha \in H^{*}$. For brevity we shall write $\Pi$ instead of $\Pi^{\mathcal{C} \# H^{*}}$

We choose a left integral $\lambda \in H^{*}$ and an element $t \in H$ such that $t \in H$ such that $\lambda<t=\varepsilon$.

Theorem 2.2. Let $\mathcal{D} \subseteq \mathcal{C}$ be an $H$-comodule category where $H$ is a finite dimensional Hopf algebra. The following statements are equivalent:

(i) The extension $\mathcal{D} \subseteq \mathcal{C}$ is $H$-Galois.

(ii) The functor $\Pi: \mathcal{C} \# H^{*} \longrightarrow \mathcal{E}\left(\mathcal{C}_{\mathcal{D}}\right)$ is an isomorphism of linear categories and ${ }_{x} \mathcal{C}_{\bullet}$ is a finitely generated projective right $\mathcal{D}$-module, for any $x \in \mathcal{C}_{0}$.

(iii) The $\operatorname{map}_{x} \gamma_{y}:{ }_{x} \mathcal{C}_{\bullet} \otimes_{\mathcal{D}} \bullet \mathcal{C}_{y} \longrightarrow{ }_{x} \mathcal{C}_{y} \# H^{*}$, defined by

$$
{ }_{x} \gamma_{y}\left(f \otimes_{\mathcal{D}} f^{\prime}\right)=(f \# \lambda)\left(f^{\prime} \# \varepsilon\right)=\sum f \circ\left(\lambda_{1} \cdot f^{\prime}\right) \# \lambda_{2},
$$

for any $f \in{ }_{x} \mathcal{C}_{u}$ and $f^{\prime} \in{ }_{u} \mathcal{C}_{y}$, is surjective.

(iv) For any Hopf module $M$ the canonical morphism $\varphi: \mathcal{C}_{\otimes_{\mathcal{D}}} M^{c o(H)} \longrightarrow M$, whose $x$-component is given by ${ }_{x} \varphi\left(f \otimes_{\mathcal{D}} m\right)=f \triangleright m$ for any $f \in{ }_{x} \mathcal{C}_{u}$ and $m \in{ }_{u} M$, is an isomorphism of Hopf modules.

Proof. We start the proof by remarking that ${ }_{x} \gamma_{y}$ satisfy the following relation:

$$
{ }_{x} \gamma_{y}=\left({ }_{x} \mathrm{C}_{y} \otimes \theta\right) \circ{ }_{x} \beta_{y},
$$

where $\theta: H \longrightarrow H^{*}$ is the $\mathbb{k}$-linear isomorphism defined by $\theta(h)=\lambda<h$ and ${ }_{x} \mathrm{C}_{y} \otimes \theta$ stands for the tensor product of the identity map of ${ }_{x} \mathrm{C}_{y}$ and $\otimes$. 
Indeed, an easy computation shows us that the above equation is equivalent to

$$
\sum f \circ\left(\lambda_{1} \cdot f^{\prime}\right) \# \lambda_{2}=\sum f \circ f_{0}^{\prime} \#\left(\lambda<f_{1}^{\prime}\right),
$$

for any $f \in{ }_{x} \mathcal{C}_{u}$ and $f^{\prime} \in{ }_{u} \mathcal{C}_{y}$. On the other hand, the equation

$$
\sum\left(\lambda_{1} \cdot f^{\prime}\right) \# \lambda_{2}=\sum f_{0}^{\prime} \#\left(\lambda \leftarrow f_{1}^{\prime}\right)
$$

clearly implies (7). Since two elements $\sum_{i=1}^{n} f_{i} \otimes \alpha_{i}$ and $\sum_{j=1}^{m} g_{j} \otimes \beta_{j}$ are equal in ${ }_{x} \mathrm{e}_{y} \otimes H^{*}$ if and only if $\sum_{i=1}^{n} \alpha_{i}(h) f_{i}=\sum_{j=1}^{m} \beta_{j}(h) g_{j}$, for all $h \in H$, the above identity follows by the computation below:

$$
\sum \lambda_{2}(h)\left(\lambda_{1} \cdot f^{\prime}\right)=\sum \lambda_{1}\left(f_{1}^{\prime}\right) \lambda_{2}(h) f_{0}^{\prime}=\sum \lambda\left(f_{1}^{\prime} h\right) f_{0}^{\prime}=\sum\left(\lambda<f_{1}^{\prime}\right)(h) f_{0}^{\prime} .
$$

In conclusion, (6) holds. Using this relation we can now prove that (i) and (iii) are equivalent. Since $\theta$ is an isomorphism, obviously (i) implies (iii). On the other hand, if we assume that (iii) holds, then ${ }_{x} \beta_{y}$ is surjective for any $x, y \in \mathcal{C}_{0}$. By Theorem 2.1 it follows that ${ }_{x} \beta_{y}$ is bijective, so $\mathcal{D} \subseteq \mathcal{C}$ is $H$-Galois.

We shall next show that (ii) implies (iii). Since ${ }_{x} \mathcal{C}_{\bullet}$ is projective and finitely generated there exist the objects $x_{i} \in \mathcal{C}_{0}$, the elements $f^{i} \in{ }_{x} \mathcal{C}_{x_{i}}$ and the morphisms $\varphi^{i}:{ }_{x} \mathcal{C}_{\bullet} \longrightarrow{ }_{x_{i}} \mathcal{D}_{\bullet}$, with $i \in\{1, \ldots, n\}$, such that (4) holds for any $f \in{ }_{x} \mathrm{C}_{u}$. As ${ }_{x_{i}} \mathcal{D}_{u} \subseteq{ }_{x_{i}} \mathrm{C}_{u}$ we can see every $\varphi^{i}$ as a morphism in $\operatorname{Hom}_{\mathcal{D}}\left({ }_{x} \mathcal{C}_{\bullet},{ }_{x_{i}} \mathrm{C}_{\bullet}\right)={ }_{x_{i}} \mathcal{E}\left(\mathcal{C}_{\mathcal{D}}\right){ }_{x}$. Since $\Pi: \mathcal{C} \# H^{*} \longrightarrow \mathcal{E}\left(\mathcal{C}_{\mathcal{D}}\right)$ is an isomorphism of $\mathbb{k}$-linear categories, there exists $\xi_{i} \in{ }_{x_{i}} \mathrm{C}_{x} \otimes H^{*}$ such that ${ }_{x_{i}} \Pi_{x}\left(\xi_{i}\right)=\varphi^{i}$. In other words, for $f \in{ }_{x} \mathrm{C}_{u}$ we have $\varphi_{u}^{i}(f)=\xi_{i} \rightarrow f$. Recall that the action of $\mathrm{C} \# H^{*}$ on ${ }_{x} \mathrm{e} \bullet$ is given by the formula

$$
\left(f^{\prime} \# \alpha\right) \rightarrow f=f^{\prime} \circ(\alpha \cdot f)
$$

for any $f^{\prime} \in{ }_{v} \mathcal{C}_{x}, f \in{ }_{x} \mathcal{C}_{u}$ and $\alpha \in H^{*}$. If we write $\xi_{i}=\sum_{j=1}^{m_{i}} f_{i j} \otimes \alpha_{i j}$, with $f_{i j}$ in ${ }_{x_{i}} \mathrm{e}_{x}$ and $\alpha_{i j}$ in $H^{*}$, it follows that for every $f \in{ }_{x} \mathcal{C}_{u}$ we have

$$
\varphi_{u}^{i}(f)=\sum_{j=1}^{m_{i}} f_{i j} \circ\left(\alpha_{i j} \cdot f\right) .
$$

We claim that

$$
\sum_{i=1}^{n} \sum_{j=1}^{m_{i}} f^{i} \circ f_{i j} \# \alpha_{i j}=1_{x} \# \varepsilon .
$$

Indeed, let $\xi$ denote the left hand side of the equation (8). By the definition of $\Pi$ and using the relation (4), for an arbitrary element $f$ in ${ }_{x} \mathcal{C}_{u}$, we obtain:

$$
{ }_{x} \Pi_{x}(\xi)(f)=\sum_{i=1}^{n} \sum_{j=1}^{m_{i}} f^{i} \circ f_{i j} \circ\left(\alpha_{i j} \cdot f\right)=\sum_{i=1}^{n} f^{i} \circ \varphi_{u}^{i}(f)=f={ }_{x} \Pi_{x}\left(1_{x} \# \varepsilon\right)(f) .
$$


It follows that ${ }_{x} \Pi_{x}(\xi)={ }_{x} \Pi_{x}\left(1_{x} \# \varepsilon\right)$, so (8) is proved as ${ }_{x} \Pi_{x}$ is isomorphism.

We also claim that $\xi_{i} \in\left({ }_{x_{i}} \mathrm{e}_{x} \otimes H^{*}\right)^{H^{*}}$, where the $H^{*}$-module structure is defined by the diagonal action. By the definition of ${ }_{x_{i}} \Pi_{x}$ and the fact that $\mathcal{C}$ is an $H^{*}$-module category, for $\alpha \in H^{*}$ and $f$ as above, we get

$$
{ }_{x_{i}} \Pi_{x}\left(\alpha \cdot \xi_{i}\right)(f)=\sum_{j=1}^{m_{i}}\left(\alpha_{1} \cdot f_{i j}\right) \circ\left[\left(\alpha_{2} * \alpha_{i j}\right) \cdot f\right]=\alpha \cdot \sum_{j=1}^{m_{i}} f_{i j} \circ\left(\alpha_{i j} \cdot f\right) .
$$

Since $\varphi_{u}^{i}(f)$ is an element in $x_{i} \mathcal{D}_{u}$ it follows that

$$
{ }_{x_{i}} \Pi_{x}\left(\alpha \cdot \xi_{i}\right)(f)=\alpha \cdot \varphi_{u}^{i}(f)=\alpha\left(1_{H}\right) \varphi_{u}^{i}(f)={ }_{x_{i}} \Pi_{x}\left(\alpha\left(1_{H}\right) \xi_{i}\right)(f) .
$$

Therefore $\alpha \cdot \xi_{i}=\alpha\left(1_{H}\right) \xi_{i}$, so our claim is proved. Since $\xi_{i}$ is $H^{*}$-invariant, by $\S 1.4$, there exists an unique $f_{i}^{\prime} \in{ }_{x_{i}} \mathrm{e}_{x}$ such that $\xi_{i}=\sum \lambda_{1} \cdot f_{i}^{\prime} \# \lambda_{2}$. Then, in view of of the definition of $\xi_{i}$ and of the equation (8) we get

$$
1_{x} \# \varepsilon=\sum_{i=1}^{n} f^{i} \circ\left(\lambda_{1} \cdot f_{i}^{\prime}\right) \# \lambda_{2}=\sum_{i=1}^{n}\left(f^{i} \# \lambda\right)\left(f_{i}^{\prime} \# \varepsilon\right) .
$$

This means that $1_{x} \# \varepsilon$ is in the image of $\gamma_{x}$. Now we can prove that every ${ }_{y} \gamma_{x}$ is surjective. Indeed, for $f \in{ }_{y} \mathrm{e}_{x}$ and $\alpha \in H^{*}$, we have

$$
f \# \alpha=(f \# \alpha)\left(1_{x} \# \varepsilon\right)=\sum_{i=1}^{n}(f \# \alpha)\left(f^{i} \# \lambda\right)\left(f_{i}^{\prime} \# \varepsilon\right) .
$$

By using the definition of the multiplication in the smash product and the fact that $\lambda$ is a left integral in $H^{*}$ we obtain the following equalities:

$$
f \# \alpha=\sum_{i=1}^{n} \sum\left[f \circ\left(\alpha_{1} \cdot f^{i}\right) \# \alpha_{2} * \lambda\right]\left(f_{i}^{\prime} \# \varepsilon\right)=\sum_{i=1}^{n}\left[f \circ\left(\alpha \cdot f^{i}\right) \# \lambda\right]\left(f_{i}^{\prime} \# \varepsilon\right) .
$$

We conclude that ${ }_{y} \gamma_{x}$ is surjective for any $x, y \in \mathcal{C}_{0}$, as

$$
f \# \alpha=\sum_{i=1}^{n}\left[f \circ\left(\alpha \cdot f^{i}\right) \# \lambda\right]\left(f_{i}^{\prime} \# \varepsilon\right)={ }_{y} \gamma_{x}\left(\sum_{i=1}^{n} f \circ\left(\alpha \cdot f^{i}\right) \otimes_{\mathcal{D}} f_{i}^{\prime}\right) .
$$

Let us show that (iii) implies (ii). Let $x \in \mathcal{C}_{0}$. Since, by hypothesis, ${ }_{x} \gamma_{x}$ is surjective for any $x \in \mathcal{C}_{0}$ there exist $f^{i} \in{ }_{x} \mathcal{C}_{x_{i}}$ and $f_{i}^{\prime} \in{ }_{x_{i}} \mathcal{C}_{x}$ such that

$$
\sum_{i=1}^{n}\left(f^{i} \# \lambda\right)\left(f_{i}^{\prime} \# \varepsilon\right)=1_{x} \# \varepsilon,
$$


where $x_{1}, \ldots, x_{n}$ are some objects in $\mathcal{C}_{0}$. In order to show that ${ }_{x} \mathcal{C}_{\bullet}$ is a finitely generated projective right $\mathcal{D}$-module, for every $i \in\{1, \ldots, n\}$, we shall construct $\varphi^{i} \in \operatorname{Hom}_{\mathcal{D}}\left({ }_{x} \mathcal{C}_{\bullet}, x_{i} \mathcal{D}_{\bullet}\right)$ such that relation (4) holds, where $u$ and $f$ are arbitrary elements in $\mathcal{C}_{0}$ and ${ }_{x} \mathcal{C}_{u}$, respectively. By definition, we take $\varphi^{i}$ to be the family of linear maps whose elements are given by

$$
\varphi_{u}^{i}(f)=\lambda \cdot\left(f_{i}^{\prime} \circ f\right) .
$$

Since $\lambda$ is a left integral, $\varphi_{u}^{i}(f) \in{ }_{x_{i}} \mathcal{D}_{u}$, so $\varphi_{u}^{i}$ is well defined. Using once again that $\lambda$ is a left integral, and the fact that $\mathcal{C}$ is an $H^{*}$-module category it results immediately that $\varphi^{i}$ is a morphism of right $\mathcal{D}$-modules. To prove the relation (4) we remark that

$$
\sum_{i=1}^{n} f^{i} \circ \varphi_{u}^{i}(f)=\sum_{i=1}^{n} f^{i} \circ\left[\lambda \cdot\left(f_{i}^{\prime} \circ f\right)\right]=\sum_{i=1}^{n} \sum f^{i} \circ\left(\lambda_{1} \cdot f_{i}^{\prime}\right) \circ\left(\lambda_{2} \cdot f\right) .
$$

On the other hand, using (9) we deduce that

$$
\sum_{i=1}^{n} \sum f^{i} \circ\left(\lambda_{1} \cdot f_{i}^{\prime}\right) \otimes \lambda_{2} \otimes f=1_{x} \otimes \varepsilon \otimes f .
$$

Since $\mathcal{C}$ is a left $H^{*}$-module, applying $\operatorname{Id}_{x} \mathfrak{e}_{x} \otimes \cdot$ to the both sides of the above equality and then using the composition of morphisms in $\mathcal{C}$, we get

$$
\sum_{i=1}^{n} \sum f^{i} \circ\left(\lambda_{1} \cdot f_{i}^{\prime}\right) \circ\left(\lambda_{2} \cdot f\right)=f .
$$

Therefore (4) holds, so we have just proved that ${ }_{x} \mathrm{C}_{\bullet}$ is a finitely generated projective $\mathcal{D}$-module.

We still have to show that ${ }_{y} \Pi_{x}:{ }_{y} \mathcal{C}_{x} \# H^{*} \longrightarrow \operatorname{Hom}_{\mathcal{D}}\left({ }_{x} \mathcal{C}_{\bullet},{ }_{y} \mathcal{C}_{\bullet}\right)$ is an isomorphism. We start by showing that each map is injective. Let $\xi=$ $\sum_{j=1}^{m} f_{j}^{\prime \prime} \# \alpha_{j}$ be an element in $\operatorname{Ker}_{y} \Pi_{x}$, where $f_{j}^{\prime \prime} \in{ }_{y} \mathcal{e}_{x}$ and $\alpha_{j} \in H^{*}$. Then $\sum_{j=1}^{m} f_{j}^{\prime \prime} \circ\left(\alpha_{j} \cdot f\right)=0$ for any $f \in{ }_{x} \mathcal{C}_{u}$. Since $\xi=\xi\left(1_{x} \# \varepsilon\right)$, and taking into account the relation (9) we get

$$
\xi=\sum_{j=1}^{m} \sum_{i=1}^{n}\left(f_{j}^{\prime \prime} \# \alpha_{j}\right)\left(f^{i} \# \lambda\right)\left(f_{i}^{\prime} \# \varepsilon\right)=\sum_{i=1}^{n} \sum_{j=1}^{m}\left[f_{j}^{\prime \prime} \circ\left(\alpha_{j} \circ f^{i}\right) \# \lambda\right]\left(f_{i}^{\prime} \# \varepsilon\right)=0 .
$$

Thus the kernel of ${ }_{y} \Pi_{x}$ is trivial. To prove that ${ }_{y} \Pi_{x}$ is surjective we pick up $\mu:{ }_{x} \mathcal{C}_{\bullet} \longrightarrow{ }_{y} \mathcal{C}_{\bullet}$, a morphism of right $\mathcal{D}$-modules. For $f \in{ }_{x} \mathcal{C}_{u}$, we have

$$
\mu_{u}(f)=\mu_{u}\left(\sum_{i=1}^{n} \sum f^{i} \circ\left[\lambda \cdot\left(f_{i}^{\prime} \circ f\right)\right]\right)=\sum_{i=1}^{n} \sum \mu_{x_{i}}\left(f^{i}\right) \circ\left(\lambda_{1} \cdot f_{i}^{\prime}\right) \circ\left(\lambda_{2} \cdot f\right),
$$


as $\mu$ is a morphism of $\mathcal{D}$-modules and $\lambda \cdot\left(f^{i} \circ f\right)=\sum\left(\lambda_{1} \cdot f^{i}\right) \circ\left(\lambda_{2} \cdot f\right)$ is an element in ${ }_{x_{i}} \mathcal{D}_{u}$. We conclude that

$$
\mu_{u}(f)={ }_{y} \Pi_{x}\left(\sum_{i=1}^{n}\left(\mu_{x_{i}}\left(f^{i}\right) \# \lambda\right)\left(f_{i}^{\prime} \# \varepsilon\right)\right)(f)
$$

so ${ }_{y} \Pi_{x}$ is a surjection.

Let us show that (iii) implies (iv). We fix a Hopf module $(M, \triangleright, \cdot)$. Here $\triangleright$ and $\cdot$ denote the actions of $\mathcal{C}$ and of $H^{*}$ on $M$, respectively. We have seen that the category of Hopf modules is isomorphic to the category of left $\mathcal{C} \# H^{*}$ modules. Therefore $M$ may be regarded as a left $\mathcal{C} \# H^{*}$-module, whose structure map will be denoted by $\rightarrow$. Hence, for $f \in{ }_{x} \mathrm{e}_{y}, \alpha \in H^{*}$ and $m \in M$, we have

$$
(f \# \alpha) \rightarrow m=f \triangleright(\alpha \cdot m) .
$$

As in the proof of the preceding implication, for a given $x$ in $\mathcal{C}_{0}$, there exist $f^{1}, \ldots, f^{n} \in{ }_{x} \mathrm{C}_{x_{i}}$ and $f_{i}^{\prime}, \ldots, f_{n}^{\prime} \in{ }_{x_{i}} \mathrm{C}_{x}$ such that equation (9) holds. Let $\xi_{i}:=\left(1_{x_{i}} \# \lambda\right)\left(f_{i}^{\prime} \# \varepsilon\right)$. Our goal is to prove that ${ }_{x} \varphi:{ }_{x} \mathrm{C}_{\bullet} \otimes_{\mathcal{D}} M^{\operatorname{co}(H)} \longrightarrow{ }_{x} M$ is invertible. Let

$$
{ }_{x} \psi:{ }_{x} M \longrightarrow{ }_{x} \mathcal{C}_{\bullet} \otimes_{\mathcal{D}} M^{c o(H)}, \quad{ }_{x} \psi(m)=\sum_{i=1}^{n} f^{i} \otimes_{\mathcal{D}}\left(\xi_{i} \rightarrow m\right) .
$$

It is easy to see that ${ }_{x} \psi$ is well defined, that is $\xi_{i} \rightarrow m \in\left({ }_{x_{i}} M\right)^{c o(H)}$, as $\lambda$ is a left integral in $H^{*}$ and $\xi_{i} \rightarrow m=\lambda \cdot\left(f_{i}^{\prime} \triangleright m\right)$.

We can now prove that ${ }_{x} \psi$ is the inverse of ${ }_{x} \varphi$. With the above notations, we have:

$$
\left({ }_{x} \varphi \circ{ }_{x} \psi\right)(m)=\sum_{i=1}^{n}{ }_{x} \varphi\left(f^{i} \otimes_{\mathcal{D}} \xi_{i} \rightarrow m\right)=\sum_{i=1}^{n} f^{i} \triangleright\left(\xi_{i} \rightarrow m\right) .
$$

By the definition of the right $\mathcal{C} \# H^{*}$-action on $M$ and using (9) we get

$$
\left({ }_{x} \varphi \circ{ }_{x} \psi\right)(m)=\sum_{i=1}^{n}\left[f^{i} \circ\left(\lambda_{1} \cdot f_{i}^{\prime}\right) \# \lambda_{2}\right] \rightarrow m=\left(1_{x} \# \varepsilon\right) \rightarrow m=m
$$

To prove that ${ }_{x} \psi \circ{ }_{x} \varphi$ is the identity of ${ }_{x} \mathcal{C}_{\bullet} \otimes_{\mathcal{D}} M^{c o(H)}$ let us pick up an element $\zeta=\sum_{j=1}^{p} f_{j}^{\prime \prime} \otimes_{\mathcal{D}} m_{j}$, with $f_{j}^{\prime \prime} \in{ }_{x} \mathcal{C}_{u_{j}}$ and $m_{j} \in{ }_{u_{j}} M^{c o(H)}={ }_{u_{j}} M^{H^{*}}$. 
Thus

$$
\begin{aligned}
\left({ }_{x} \psi \circ{ }_{x} \varphi\right)(\zeta) & \stackrel{(A)}{=} \sum_{i=1}^{n} \sum_{j=1}^{p} f^{i} \otimes_{\mathcal{D}}\left[\xi_{i} \rightarrow\left(f_{j}^{\prime \prime} \triangleright m_{j}\right)\right] \\
& \stackrel{(B)}{=} \sum_{i=1}^{n} \sum_{j=1}^{p} f^{i} \otimes_{\mathcal{D}}\left[(1 \# \lambda)\left(f_{i}^{\prime} \# \varepsilon\right)\left(f_{j}^{\prime \prime} \# \varepsilon\right)\right] \rightarrow m_{j} \\
& \stackrel{(C)}{=} \sum_{i=1}^{n} \sum_{j=1}^{p} \sum f^{i} \otimes_{\mathcal{D}}\left[\left(\lambda_{1} \cdot f_{i}^{\prime}\right) \circ\left(\lambda_{2} \cdot f_{j}^{\prime \prime}\right) \# \lambda_{3}\right] \rightarrow m_{j} \\
& \stackrel{(D)}{=} \sum_{i=1}^{n} \sum_{j=1}^{p} \sum f^{i} \otimes_{\mathcal{D}}\left[\left(\lambda_{1} \cdot f_{i}^{\prime}\right) \circ\left(\lambda_{2} \cdot f_{j}^{\prime \prime}\right)\right] \triangleright\left(\lambda_{3} \cdot m_{j}\right) \\
& \stackrel{(E)}{=} \sum_{i=1}^{n} \sum_{j=1}^{p} \sum f^{i} \otimes_{\mathcal{D}}\left[\left(\lambda_{1} \cdot f_{i}^{\prime}\right) \circ\left(\lambda_{2} \cdot f_{j}^{\prime \prime}\right)\right] \triangleright m_{j} \\
& \stackrel{(F)}{=} \sum_{i=1}^{n} \sum_{j=1}^{p} f^{i} \otimes_{\mathcal{D}}\left[\lambda \cdot\left(f_{i}^{\prime} \circ f_{j}^{\prime \prime}\right)\right] \triangleright m_{j} \\
& \stackrel{(G)}{=} \sum_{i=1}^{n} \sum_{j=1}^{p} f^{i} \circ\left[\lambda \cdot\left(f_{i}^{\prime} \circ f_{j}^{\prime \prime}\right)\right] \otimes_{\mathcal{D}} m_{j} \\
& \stackrel{(H)}{=} \sum_{i=1}^{n} \sum_{j=1}^{p} \sum^{i} f^{i}\left(\lambda_{1} \cdot f_{i}^{\prime}\right) \circ\left(\lambda_{2} \cdot f_{j}^{\prime \prime}\right) \otimes_{\mathcal{D}} m_{j} \\
& \stackrel{(I)}{=} \sum_{j=1}^{p} f_{j}^{\prime \prime} \otimes_{\mathcal{D}} m_{j}=\zeta .
\end{aligned}
$$

Let us briefly explain the above computation. The relations (A) and (B) follows by the definition of the map ${ }_{x} \psi$ and of the element $\xi_{i}$, respectively. The relations (C) and (D) are consequences of the definition of the composition of morphisms in $\mathrm{C} \# H^{*}$ and of the $\mathfrak{C} \# H^{*}$-module structure on $M$. By using the fact that $m_{j} \in{ }_{u_{j}} M^{c o(H)}$ we obtain (E). The relations (F) and (H) hold as $\mathcal{C}$ is an $H^{*}$-module category. For $(\mathrm{G})$ one uses the observation that $\lambda \cdot v$ is $H^{*}$ invariant, for any left integral $\lambda \in H^{*}$ and any element $v$ in a left $H^{*}$-module $V$. For a fixed $j$, tensorising both sides of (9) to the right with $f_{j}^{\prime \prime}$ we obtain

$$
\sum_{i=1}^{n} f_{i} \circ\left(\lambda_{1} \cdot f_{i}^{\prime}\right) \otimes \lambda_{2} \otimes f_{j}^{\prime \prime}=1_{x} \otimes \varepsilon \otimes f_{j}^{\prime \prime} .
$$


Using the $H^{*}$-module structure of ${ }_{x} \mathfrak{C}_{u_{j}}$ and then the composition in $\mathcal{C}$, we get

$$
\sum_{i=1}^{n} f_{i} \circ\left(\lambda_{1} \cdot f_{i}^{\prime}\right) \circ\left(\lambda_{2} \cdot f_{j}^{\prime \prime}\right)=1_{x} \circ f_{j}^{\prime \prime}=f_{j}^{\prime \prime} .
$$

Thus (I) holds true as well. To finish the proof of this implication we have to show that $\varphi$ is an isomorphism of $\mathfrak{C} \# H^{*}$-modules. Indeed, for $f \in{ }_{x} \mathfrak{C}_{u}$, $f^{\prime} \in{ }_{u} \mathrm{e}_{v}, \alpha \in H^{*}$ and $m \in{ }_{v} M$ we have:

$$
{ }_{x} \varphi\left((f \# \alpha) \rightarrow\left(f^{\prime} \otimes_{\mathcal{D}} m\right)\right)={ }_{x} \varphi\left(\left[(f \# \alpha) \rightarrow f^{\prime}\right] \otimes m\right)=\left[f \circ\left(\alpha \cdot f^{\prime}\right)\right] \triangleright m .
$$

On the other hand, as $M$ is a Hopf module and $m$ is $H^{*}$-invariant, we have:

$$
(f \# \alpha) \rightarrow{ }_{u} \varphi\left(f^{\prime} \otimes m\right)=\sum f \triangleright\left(\alpha_{1} \cdot f^{\prime}\right) \triangleright\left(\alpha_{2} \cdot m\right)=\left[f \circ\left(\alpha \cdot f^{\prime}\right)\right] \triangleright m,
$$

so our claim has been proved.

It remains to show that (iv) implies (iii). We have to prove that ${ }_{x} \gamma_{y}$ is surjective for all $x \in \mathcal{C}_{0}$. Proceeding as in the proof of the implication (ii) $\Rightarrow$ (iii) it is sufficient to show that $1_{x} \otimes \varepsilon$ belongs to the image of ${ }_{x} \gamma_{x}$. For this, we consider the left $\mathfrak{C} \# H^{*}$-module $\bullet \mathcal{C}_{x} \# H^{*}$, whose action is induced by the composition of morphisms in the smash product category. Let us denote it by $(M, \rightarrow)$. By assumption, the components of the morphism $\varphi$ : $\mathcal{C} \otimes_{\mathcal{D}} M^{c o(H)} \longrightarrow M$ are bijective. Thus $1_{x} \otimes \varepsilon \in{ }_{x} M$ is in the image of ${ }_{x} \varphi$, so there exist $f_{1}, \ldots, f_{n} \in{ }_{x} \mathfrak{C}_{x_{i}}$ and $m_{1}, \ldots, m_{n} \in{ }_{x_{i}} M^{c o(H)}$ such that

$$
\sum_{i=1}^{n} f_{i} \triangleright m_{i}=1_{x} \# \varepsilon .
$$

On the other hand we saw that every $H^{*}$-invariant element in ${ }_{y} \mathfrak{C}_{x} \otimes H^{*}$ is of the form $(1 \otimes \lambda)\left(f^{\prime} \otimes \varepsilon\right)$, with $f^{\prime} \in{ }_{y} \mathcal{C}_{x}$. Then there exist $f_{i}^{\prime} \in{ }_{x_{i}} \mathrm{e}_{x}$ such that

$$
m_{i}=\left(1_{x_{i}} \# \lambda\right)\left(f_{i}^{\prime} \# \varepsilon\right)=\sum \lambda_{1} \cdot f_{i}^{\prime} \# \lambda_{2} .
$$

Therefore,

$$
1_{x} \# \varepsilon=\sum_{i=1}^{n} f_{i} \triangleright m_{i}=\sum_{i=1}^{n} f_{i} \circ\left(\lambda_{1} \cdot f_{i}^{\prime}\right) \# \lambda_{2}={ }_{x} \gamma_{x}\left(\sum_{i=1}^{n} f_{i} \otimes f_{i}^{\prime}\right),
$$

so the theorem is proved.

Corollary 2.3. Let $H$ be a Hopf algebra of dimension $n$. If $\mathcal{C}=\mathcal{D} \#_{\sigma} H$ is a cross product with invertible cocycle then

$$
\left(\mathcal{D} \#{ }_{\sigma} H\right) \# H^{*} \simeq M_{n}(\mathcal{D}) .
$$


Proof. By [14, Theorem 3.6 and Theorem 3.9] the extension $\mathcal{D} \subseteq \mathcal{C}$ is $H$ Galois. In view of the previous theorem, $\mathcal{C} \# H^{*} \stackrel{\simeq}{\longrightarrow} \mathcal{E}\left(\mathcal{C}_{\mathcal{D}}\right)$. Since $H$ is finite dimensional and $\sigma$ is invertible in convolution it follows that ${ }_{x} \mathrm{e}_{\bullet}$ is a $\mathcal{D}$-module isomorphic with ${ }_{x} \mathcal{D}_{\bullet}^{n}$, see $\S 1.2$. Hence, by $\S 1.6$, we conclude that $\mathcal{E}\left(\mathcal{C}_{\mathcal{D}}\right) \simeq$ $M_{n}(\mathcal{D})$, where $M_{n}(\mathcal{D})$ is the category of square matrix of order $n$ with elements in $\mathcal{D}$.

\section{References}

[1] J. Bergen and S. Montgomery, Smash products and outer derivations, Israel J. Math. 53 (1986), no. 3, 321-345.

[2] R. J. Blattner and S. Montgomery, Crossed products and Galois extensions of Hopf algebras, Pacific J. Math. 137 (1989), no. 1, 37-54.

[3] C. Cibils and A. Solotar, Galois coverings, Morita equivalence and smash extensions of categories over a field, Doc. Math. 11 (2006), 143-159.

[4] M. Cohen, D.Fischman and S. Montgomery, Hopf Galois extensions, smash products, and Morita equivalence, J. Algebra 133 (1990), no. 2, 351-372.

[5] M. Cohen and S. Montgomery, Group-graded rings, smash products, and group actions, Trans. Amer. Math. Soc. 282 (1984), 237-258.

[6] S. Dăscălescu, C. Năstăsescu and Ş. Raianu, Hopf algebras. An introduction, Monographs and Textbooks in Pure and Applied Mathematics, vol. 235, Marcel Dekker Inc., New York, 2001.

[7] E. Herscovich and A. Solotar, Hochschild-Mitchell Cohomology and Galois Extensions, J. Pure Appl. Algebra 209 (2007), no. 1, 37-55.

[8] E. Herscovich and A. Solotar, Derived invariance of Hochschild-Mitchell (co)homology and one-point extensions, J. Algebra 315 (2007), no. 2, 852-873.

[9] H. Kreimer and M. Takeuchi, Hopf algebras and Galois extensions of an algebra, Indiana Univ. Math J. 30 (1981), 675-692.

[10] B. Mitchell, Rings with several objects, Advances in Math. 8 (1972), 1-161.

[11] S. Montgomery, Hopf algebras and their actions on rings, CBMS Regional Conference Series in Mathematics, vol. 82, Published for the Conference Board of the Mathematical Sciences, Washington, DC, 1993. 
[12] Y. Nakagami and M. Takesaki, Duality for crossed products of Von Newmann algebras, Lecture Notes in Mathematics, vol. 731 (1979), Springer, Berlin, 1979.

[13] H.-J. Schneider, Normal basis and transitivity of crossed products for Hopf algebras, J. Algebra 152 (1992), no. 2, 289-312.

[14] A. Stănescu and D. Ştefan, Cleft comodule categories, to appear in Comm. Algebra.

Anca STĂNESCU,

Department of Mathematics,

University of Bucharest,

14 Academiei Street, Ro-010014, Bucharest, Romania.

Email: ancastanescu2002@yahoo.com 